\title{
Localisation of antigens in the gut post-challenge with Streptococcus iniae in vaccinated and non-vaccinated red hybrid tilapia (Oreochromis sp.)
}

\begin{abstract}
This study was conducted to determine the localisation of antigen in the gut of vaccinated and non-vaccinated red hybrid tilapia post-challenge via immunohistochemistry and scanning electron microscopy. All groups (1, 2, 3, and 4) were vaccinated orally with feed-based killed whole-cells of Streptococcus iniae (FKV). Group 1 was fed with the FKV for 3 days consecutively only on day 0 ; group 2 was fed with the FKV for 3 days consecutively on day 0 and booster on day 14; group 3 was fed with the FKV for 3 days consecutively on day 0 , followed by a booster on day 14 and 21; and group 4 was fed with the FKV for 5 days consecutively on day 0 , followed by a booster on day 14 and 21 . The remaining groups, group 5 (negative control group) and group 6 (positive control group), only fed with a commercial feed. Five fish from each group were sacrificed on a weekly basis for the entire 6 weeks for a collection of fresh small intestinal samples including post-challenge starting from 4 to $72 \mathrm{~h}$. Immunohistochemical staining was conducted using the avidin-biotin-peroxidase complex method. Scanning electron microscopy was also used to view the hindgut samples from each group. Positive control group recorded significantly the highest $(\mathrm{p}<0.05)$ antigen positivity, followed by groups 1 and 2 , meanwhile, lowest positivity in groups 3 and 4 at $12 \mathrm{~h}, 24 \mathrm{~h}$, and $72 \mathrm{~h}$, respectively. Immunohistochemistry and scanning electron microscopy showed the presence of antigens in red hybrid tilapia hindgut, protruding in goblet cells, gut-associated lymphoid tissues, and villi dome epithelium in different hours of post-challenge. Significantly, boosted group 4 with a low number of antigens in $72 \mathrm{~h}$ showed the stimulation and irregular projection of M-cells with variable morphology and antigen distribution pattern.
\end{abstract}

Keyword: Streptococcus iniae; Oral; Feed-based killed vaccine; Red hybrid tilapia (Oreochromissp.); Immunohistochemical staining; Scanning electron microscopy 